\title{
Metatranscriptomics and absolutely amplicon sequencing reveal the potentially adaptive mechanism of microbes in the deoxygenated Bohai Sea
}

Yu Han ${ }^{1}$, Mu Zhang ${ }^{1}$, Weidong Zhai ${ }^{2}$, Xiaofeng Chen ${ }^{1}$, Nianzhi Jiao ${ }^{1 *}$, Kai Tang ${ }^{1 *}$

${ }^{1}$ State Key Laboratory of Marine Environmental Science, Fujian Key Laboratory of Marine Carbon Sequestration, College of Ocean and Earth Sciences, Xiamen University, Xiamen 361102, PR China

${ }^{2}$ Institute of Marine Science and Technology, Shandong University, Qingdao 266000, PR China

* Corresponding: tangkai@xmu.edu.cn and jiao@xmu.edu.cn

Marine deoxygenation caused by eutrophication occurs continually in coastal zones, and alters microbial variety and energy shunt. Previous studies showed that $16 \mathrm{~S}$ rRNA Thaumarchaeota widely distributed in marine environments, including the costal hypoxic zones, however, their activity is still unexplored. Here, by using metatranscriptomics and absolute quantification of 16S rRNA genes approaches, we investigated the microbial activity and composition in deoxygenated water column of Bohai Sea in summer. Our results indicated that dissolved oxygen (DO) largely controls their activity and composition. As DO decreasing, the transcriptional activity of Thaumarchaeota increased significantly with invariable absolute abundance, while the transcripts of photoautotroph Cyanobacteria decreased significantly. Biofunctional analysis showed transcripts of Thaumarchaeota were preferential assigned in clusters of orthologous gene (COG) "Energy production and conversion", containing ammonia monooxygenase subunits $\mathrm{A}, \mathrm{B}, \mathrm{C}$ and Ferredoxin-related proteins. Additionally, the expression of NO-forming nitrite reductase family enzymes and metabolism of archaeal carbon fixation (3hydroxypropionic acid / 4-hydroxybutyric acid cycle) were also enhanced in low DO water. In contrast, Proteobacteria, the dominated bacterial group in our study area, showed a comparable abundance and transcripts throughout water column, and performed for COG "Translation, ribosomal structure and biogenesis" and "Posttranslational modification, protein turnover, chaperones". Comparatively, such results implied an intensified chemoautotrophic carbon fixation pathway for Thaumarchaeota in hypoxia zones, emphasizing their global ecological role.

Key words: Marine deoxygenation, microbes, metatranscriptomics 